\title{
Fractional Order Impedance Models as Rising Tools for Quantification of Unconscious Analgesia
}

\author{
Amélie Chevalier ${ }^{1}$, Dana $\operatorname{Copot}^{1}$, Clara M. Ionescu ${ }^{1}$ and Robin De Keyser ${ }^{1}$
}

\begin{abstract}
This research focuses on modeling the diffusion process that occurs in the human body when an analgesic drug is taken up, by using fractional-order impedance models (FOIMs). We discuss the measurement of a suitable feedback signal that can be used in a model-based control strategy. With this knowledge an early dawn concept of a pain sensor is presented. The major challenges that are encountered during this development consist of identification of the patient model, validation of the pain sensor and validation of the effect of the analgesic drug.

Index Terms - Analgesia, pain relief level, non-invasive pain sensor, model-based predictive control (MPC), fractional-order impedance model (FOIM)
\end{abstract}

\section{INTRODUCTION}

The last few decades, modern medicine has successfully been influenced by advanced control technologies resulting in applications such as robotic surgery, electrophysiological system life support and image-guided therapy and surgery [1]. An interesting application of control in medicine is clinical pharmacology and in particular the control of general anesthesia during surgery and in the intensive care unit (ICU). Monitoring and controlling the depth of anesthesia for surgical patients poses interesting challenges to the control engineer [2] as it is a multivariable interaction process that has captured the attention of engineers and clinicians already decades ago [3]. The first designs were expert systems that advised the anesthesiologist upon optimal drug infusion rates during clinical trials [4]. Control of anesthesia has a manifold of challenges, with multi-variable characteristics, different dynamics depending on anesthetic substances and stability problems [5].

General anesthesia, where the patient is completely unconscious, has the aim of ensuring sleep, amnesia, loss of pain, relaxation of skeletal muscles and loss of control of reflexes of the autonomic nervous system. It consists of three components acting simultaneously on the patient's vital signs: hypnosis (ensuring sleep and amnesia), analgesia (ensures loss of pain) and neuromuscular blockade (relaxes the skeletal muscles and the motor reflexes). Hypnosis is relatively well-characterized and is in standard clinical practice monitored by sensors based on electroencephalogram (EEG) data. Neuromuscular blockade immobilizes the patient during surgical procedures or intensive care and is also

\footnotetext{
${ }^{1}$ The authors are with the Department of Electrical Energy, Systems and Automation, Ghent University, Technologiepark 913, 9052 Gent, Belgium, e-mail: \{Amelie.Chevalier, Dana.Copot, ClaraMihaela.Ionescu, Robain.DeKeyser\}@UGent.be
}

a relatively well-characterized process with standard sensors, such as motion sensors, available. By contrast, analgesia is far from being well-characterized and no sensor is available for measuring the pain relief levels that a patient experiences during general anesthesia.

The advantage of automated closed loop control of anesthesia is that it gives a continuous drug delivery, contrary to intermittent control which is nowadays standard practice. A continuous drug delivery ensures that there is no underor over-dose of hypnotic or analgesic drugs that could result in patients that feel pain during surgery but are unable to move. Erroneous feedback information, biased either by the presence of artifacts (e.g. eye movement, leg movement, coughing, sneezing, choking, shivering) either by patient model mismatch, is one of the major problems for the control algorithms [6]. As result the quality of the measured signals decreases, leading to the need of complex numerical filtering techniques. The latter require longer computation times, hence introducing artificial time delays which vary from one time instant to another, dependent on the signal quality. If not dealt-with appropriately, such varying timedelays are a source of poor feedback control. Advanced control techniques such as model-based predictive control (MPC) can deal successfully with these variable time delays, nonlinearities, input and output constraints [7].

The research presented in this paper merges classical control theory with the young promising field of fractionalorder modeling to measure pain relief levels in an unconscious patient and initiate the development of a biosensor for analgesia levels. Few pioneering attempts to measure the analgesic component of general anesthesia have shown that current state-of-art is unable to deliver suitable signals and models for optimal regulation. The result is then a high risk of drug over- or under-dosing and unwanted post-operatively effects, leading to increased hospitalization and health-care costs for both society and patient [8]. We propose to employ a mathematical tool called fractional-order impedance model (FOIM) to model the pharmacological diffusion process that takes place when the human body takes up an analgesic drug such as remifentanil. These models can be used in a MPC context to control the depth of analgesia in the unconscious patient.

The paper is structured as follows: in section II, we describe analgesia and the coinciding diffusion process. Section III discusses the control method that will be used in combination with the proposed analgesia sensor and the 
possible models used in this control. The grand challenges in the development of the sensor are discussed in section IV and the conclusions are summarized in a final section.

\section{ANALGESIA AS INTEGRATED PART OF GENERAL ANESTHESIA}

General or complete anesthesia refers to inhibition of sensory, motor and sympathetic nerve transmission at the level of the brain, resulting in unconsciousness and lack of sensation. It consists of three components: hypnosis, analgesia and neuromuscular blockade. Hypnosis is a general term indicating unconsciousness and absence of post operative recall of events occurred during surgery. The level of hypnosis is related with the infusion of hypnotic drugs such as propofol and can be monitored by BIS monitoring. Analgesia is defined as an insensibility to pain without loss of consciousness. It is a state in which painful stimuli are not perceived or interpreted as pain and is usually induced by a drug, although trauma or disease may also result in a general or regional analgesia. Neuromuscular blockade is induced to prevent unwanted movement or muscle tone and causes paralysis during surgical procedures. The muscle relaxants are given intravenously (through the bloodstream) and act directly on the muscles.

Hence, analgesia is the amount of pain relief achieved during general anesthesia. The pain relief is obtained by administrating an analgesic drug such as remifentanil to the patient. The effectiveness of the analgesic drugs relies on how they are able to block the neural messages to the brain that are sent by the pain receptors. In the next sections we discuss this process of pain perception and how the analgesic drug is absorbed by the human body i.e. the drug diffusion process.

\section{A. Pain perception}

Pain perception is the complicated process where a pain signal is sent from the pain receptors found in the skin to the brain where the signal is interpreted as pain. The signal is transmitted via neurons and synapses, through the spinal cord and then to the brain.

Neurons are the basic cells in the central nervous system (CNS). Classical neurons consist of a cell body, dendrites and axons (see figure 1). After the dendrites receive the

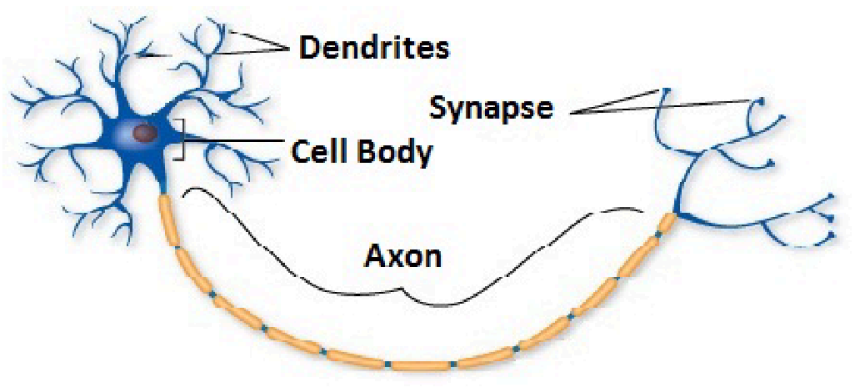

Fig. 1. Main parts of a classical neuron.

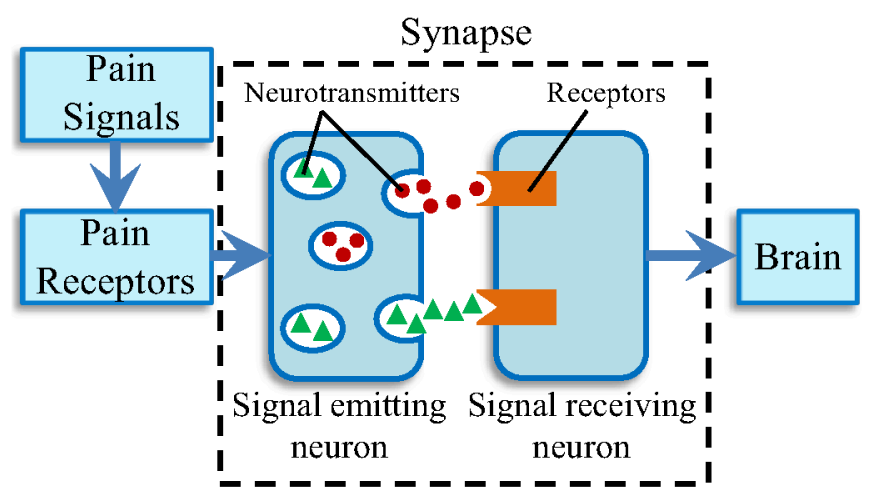

Fig. 2. Inner mechanisms of a synapse.

information from the previous cell, the axon generates an action potential (AP), which is an electrical signal, and sends this signal to the next cell via the presynaptic terminals. The presynaptic terminal forms the synapse together with the dendrites of the following cell that receive the information sent through the neuron.

A synapse is the place where two neurons communicate with each other. At this point in the communication we do not have an electrical signal anymore but a chemical signal. The chemicals used in this communication are called neurotransmitters. In figure 2 we can see a schematic of a synapse between two neurons. When the electric signal reaches the synapse at the side of the signal emitting neuron, it causes the release of chemical messengers i.e. neurotransmitters from storage vesicles. The neurotransmitters travel across a minute gap between the cells and then interact with protein molecules i.e. receptors located in the membrane surrounding the signal receiving neuron. This interaction causes biochemical reactions that result in the generation of a new electrical signal, depending on the type of neuron, neurotransmitter, and receptor involved. Each receptor has a corresponding neurotransmitter. Receptors function much like gates that enable pain signals to pass through and onto the neighboring cells.

Pain receptors, also called nociceptors, are sensory neurons that are found in any area of the body that can sense pain either externally or internally. An external example are the nociceptors in the top layer of the skin (see figure 3). Internal nociceptors are present in a variety of organs, such as the muscle, joint, bladder, gut and continuing along the digestive tract. Nociceptors can be triggered by exceeding a high threshold that has been reached by either chemical, thermal, or mechanical environments.

Afferent neurons (such as nociceptors), which send information to the CNS, travel back to the spinal cord where they form synapses in its dorsal horn. From the dorsal horn the information is then sent to the thalamus which is located near the brain. The information is then processed in the ventral posterior nucleus and sent to the cerebral cortex, the headquarters for complex thoughts. This is where the 
signals are interpreted as pain. This entire process of pain transmission is called nociception.

There are many different neurotransmitters in the human body acting in various combinations to produce painful sensations in the body. Some chemicals govern mild pain sensations while others control intense or severe pain. When tissues become injured or inflamed, chemicals are released making nociceptors much more sensitive causing them to transmit pain signals in response to even gentle stimuli such as breeze or a caress. This condition is called allodynia; a state in which pain is produced by harmless stimuli. This can be a major cause for over-dosing of analgesics and should be well-understood in order to be avoided.

\section{B. Diffusion process}

An analgesic drug interacts with the CNS to stop the communication between the nociceptors and the brain so that pain can not be perceived anymore. However, this drug needs first to be taken up by the human body. This is achieved by a complex diffusion process across various cell membranes.

An important function of a biological cell membrane is to serve as a barrier to the outside world. However, membranes are not impenetrable walls. Nutrients must be able to enter the cell and waste products have to leave in order for the cell to survive. For this and many other reasons, it is crucial that membranes be selectively permeable. For example, the movement of ions across membranes is important in regulating vital cell characteristics such as cellular $\mathrm{pH}$ and osmotic pressure [9]. Membrane permeability is also a key determinant in the effectiveness of drug absorption, distribution and elimination. For example, a drug taken orally that targets cells in the CNS must cross several membranes: first the barrier presented by the intestinal epithelium, then the walls of the capillaries that perfuse the gut, then the blood-brain barrier. Some endogenous substances and many drugs easily diffuse across the lipid bilayer. However, the lipid bilayer presents a formidable barrier to larger and

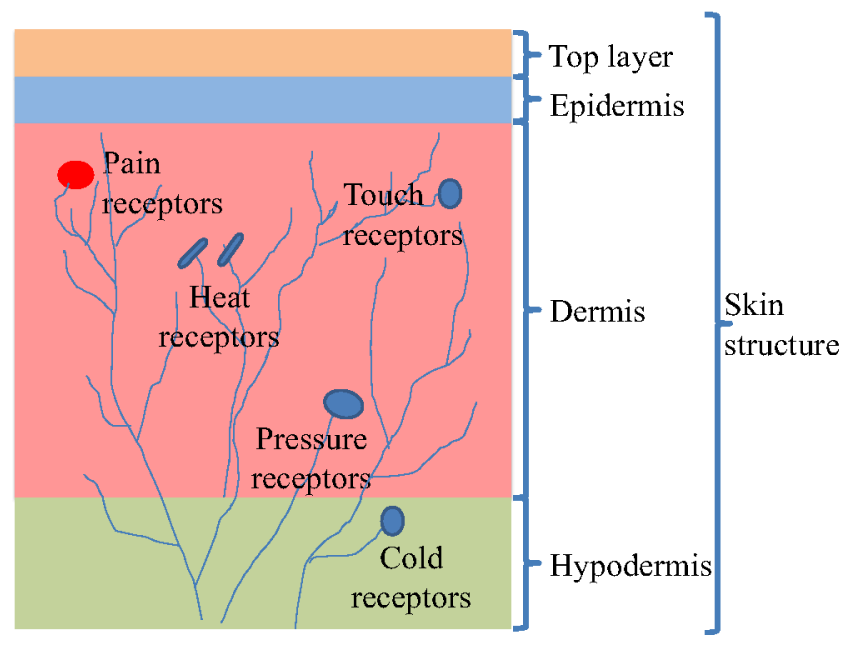

Fig. 3. Schematic overview of pain receptors in the skin. more hydrophilic molecules (such as ions). These substances must be transported across the membrane by special protein channels.

Many drugs need to pass through one or more cell membranes (see figure 4) to reach their site of action. A common feature of all cell membranes is a phospholipid bilayer, about $10 \mathrm{~nm}$ thick. Spanning this bilayer or attached to the outer or inner leaflets are glycoproteins, which may act as ion channels, receptors, intermediate messengers (G-proteins) or enzymes. Cells absorb molecules and ions from the extracellular fluid, creating a constant in- and outflow. The interesting thing about cell membranes is that relative concentrations and phospholipid bilayers prevent essential ions from entering the cell. Therefore, in order for drugs to move across the membrane these problems must be addressed. In general, this is completed by facilitated diffusion or active transport. In facilitated diffusion, relative concentrations are used to transport in and out. Active transports use energy, such as ATP (AdenosineTriPhosphate), to transfer molecules and ions in and out of the cell. Cellular signals cross the membrane through a process called signal transduction. This three-step process proceeds when a specific message encounters the outside surface of the cell and makes direct contact with a receptor. A receptor is a specialized molecule that takes information from the environment and passes it throughout various parts of the cell. Next, a connecting switch molecule, called a transducer, passes the message inwards, closer to the cell. Finally, the signal gets amplified, therefore causing the cell to perform a specific function. These functions can include moving, producing more proteins, or even sending out more signals [10].

Diffusion across the lipid bilayer is the spontaneous process where certain molecules can slip between the lipids in the bilayer and cross from one side to the other since the membranes are held together by weak forces (see figure 5). This process allows molecules that are small and lipophilic (lipid-soluble), including most drugs, to easily enter and exit cells. In order to be able to develop a sensor to measure the analgesic component of general anesthesia, we need to find

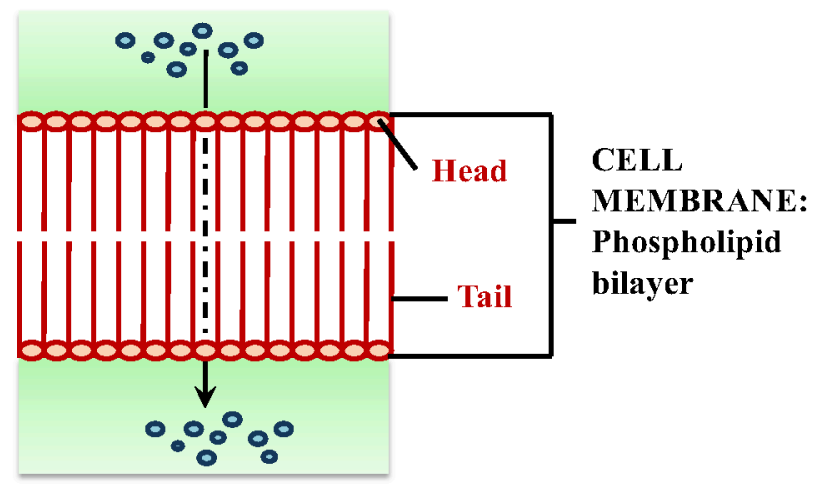

Fig. 4. Diffusion of analgesic drug through the phospholipid bilayer of the cell membrane. 


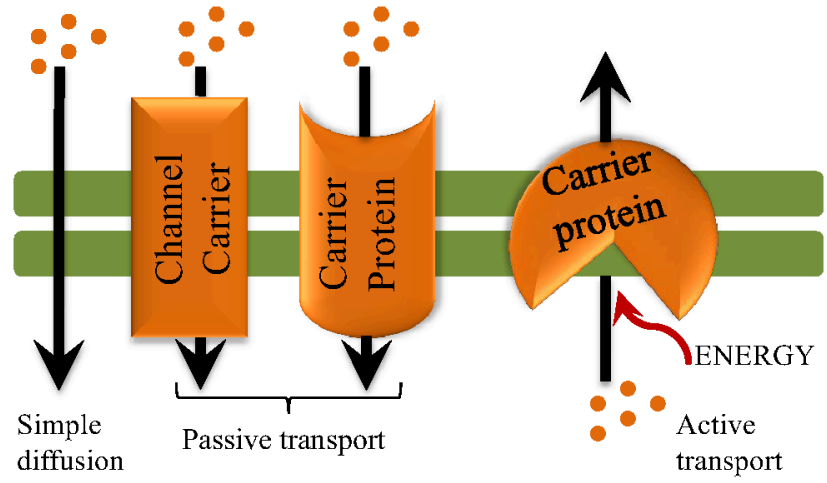

Fig. 5. Various mechanisms of diffusion of analgesic drugs across the cell membrane.

a way to model this diffusion process.

\section{AUTOMATED REGULATION OF DEPTH OF ANESTHESIA}

Nowadays, to optimally control the depth of the anesthesia, there is a need for a sensor that can measure the level of analgesia. The nonlinear response profile and inter- and intrapatient variation of the patient's analgesic state to infusion of remifentanil should be handled by a robust controller. From a clinical point-of-view, an ideal controller would guide the induction of anesthesia in order to reach the target as fast as possible without initial overshoot. Afterwards, the controller would simply maintain the desired target as well as possible. Therefore, from control engineering viewpoint, model-based predictive control plays a crucial role in solving such complex problems.

The term model-based predictive control refers to a class of computer-based control strategies. They utilize in realtime an explicit process model to predict at each control interval the future response of the controlled system. The type of models which are currently used in real-life applications are either linear dynamical system models (step response models, transfer function models, linear state space models), either nonlinear dynamical system models. The roots for MPC are dating back to around 1980, when some pioneering institutions started to develop the main ideas and computer algorithms [11]. The MPC strategy can be visualized by the block-scheme in figure 6.

\section{A. Non-invasive sensor}

General anesthesia (sedation) is difficult to assess in terms of adequacy because of its subjective nature. Several objective sedation scales such as the Ramsay Sedation Scale and the Sedation-Agitation Scale have been developed [12]. The Ramsay scoring system is one of the most commonly used scales. Even though it is simple, it can not effectively measure the quality or amount of sedation and has never been objectively validated. Newer sedation scales are reported to show improvements in validity and reliability [13]. Unfortunately, clinical sedation scores do not prevent under- or over-

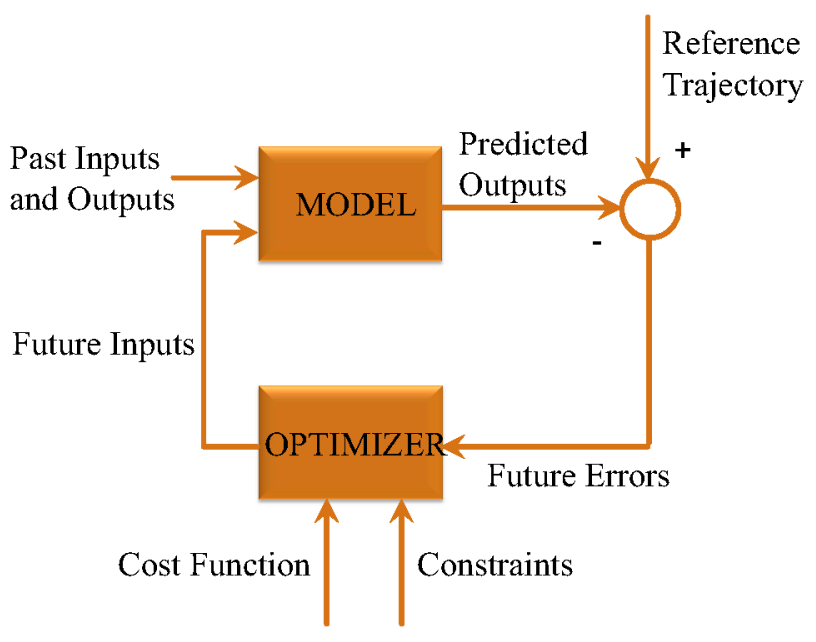

Fig. 6. An overview of the MPC strategy.

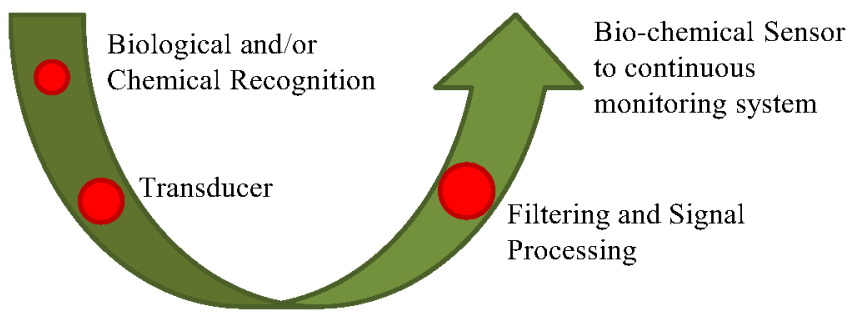

Fig. 7. three main parts of the proposed biosensor.

sedation and demand continuous bedside clinical scoring, a task performed by an alert clinical nurse sitting next to the patient.

In order to obtain a correct level of sedation in the patient, continuous monitoring of analgesia (pain relief) is of paramount importance. This demands stand-alone integrated monitoring tools for analgesia. While instrumental tools for the hypnotic component of anesthesia are standardly available and reliable (e.g. the BIS Monitor from Aspect Medical [14]), there is a serious lack of available tools to measure the analgesic component in an objective, reliable manner. Hitherto, there exists no sensor that evaluates objectively and continuously the pain relief levels during general anesthesia. The challenge originates from the fact that pain perception in the neural dynamics, and hence in the subsequent biological feedback, is not understood properly since models to characterize this complex biological process are not available.

To date there exist no integrated pain sensor and no information is available on how exactly these nociceptors can be detected. An early dawn conceptual picture of the sensor setup by means of several detection and processing steps is given in figure 7. The three main parts of a biosensor are presented: the role of biological recognition elements (receptors, enzymes, antibodies, etc.) is to differentiate the target molecules in the presence of various chemicals, the 
transducer (electrochemical, optical, magnetic, etc.) converts the bio-recognition event into a measurable signal, the signal processing part converts the signal into a readable form.

\section{B. A prediction model for $M P C$}

MPC is a model-based control strategy. Standard models include step response models, transfer function models and linear state space models, however, these models do not suffice in modeling the dynamics of the diffusion processes that occur in the human body.

In the past many attempts to model the diffusion process have been made. Fractional Order Impedance Models (FOIMs) have been shown to well-characterize these diffusion processes [15], which in essence take place ubiquitously in our body. It is therefore natural to choose these tools in detecting, understanding and characterizing the process of pain reception at the level of nociceptors.

In medicine, the field of fractional-order calculus has barely been explored. However, this research field promises to serve a whole range of applications with a large impact on the progress of science and welfare. The last decades have shown an increased interest in the research community to employ parametric model structures of fractional-order for analyzing nonlinear biological systems. The concept of fractional-order (FO) - or non-integer order - systems refers to those dynamical systems whose model structure contains arbitrary order derivatives and/or integrals [16]. The fractional-order derivatives and integrals are tools of the Fractional Calculus theory. The dynamical systems whose model can be approximated in a natural way using FO terms, exhibit specific features: viscoelasticity, diffusion and fractal structure. From previous work [17], we know now that the multiple scale adaptation of neurons is consistent with fractional order differentiation, such that the neuron's firing rate is a fractional derivative of slowly varying stimulus parameters [18]. The findings of scale-free fluctuations in the activity of neurons and synapses have been used to illustrate the existence of multiple time-scale dynamics in neurons and synapses. Additionally, there has been shown that phase-locking phenomena can be explained by the presence of fractal electrical neuronal networks, which lead to a fractional-order impedance model of the neural network [19]. However, the theoretical concepts of fractals, chaos and multi-scale analysis have not yet been employed in the field of anesthesia, where the electrical activity of the brain is altered by the effects of hypnotic (propofol) and analgesic (remifentanil) drugs.

Another option to model the diffusion processes in the human body is to use compartmental models in combination with fractional-order derivatives [20]. Three compartments are used in this diffusion model: blood, muscle and fat.

\section{Principles of Fractional Calculus}

The fractional calculus is a generalization of integration and derivation to non-integer (fractional) order operators. At first, we generalize the differential and integral operators into one fundamental operator $D_{t}^{n}$ ( $n$ the order of the operation) which is known as fractional calculus.

Several definitions of this operator have been proposed. All of them generalize the standard differential-integral operator in two main groups: (a) they become the standard differential-integral operator of any order when $n$ is an integer; (b) the Laplace transform of the operator $D_{t}^{n}$ is $s^{n}$ (provided zero initial conditions), and hence the frequency characteristic of this operator is $(j \omega)^{n}$. The latter is very appealing for the design of parametric modelling and control algorithms by using specifications in the frequency domain.

A fundamental $D_{t}^{n}$ operator, a generalization of integral and differential operators (differintegration operator), is introduced as follows:

$$
D_{t}^{n}=\left\{\begin{array}{cc}
\frac{d^{n}}{d t^{n}}, & n>0 \\
1, & n=0 \\
\int_{0}^{t}(d \alpha)^{-n}, & n<0
\end{array}\right\}
$$

where $n$ is the fractional order and $d \alpha$ is a derivative function. Since this paper will focus on the frequencydomain approach for FO derivatives and integrals, we shall not introduce the complex mathematics for time domain analysis. The Laplace transform for integral and derivative order $n$ are, respectively:

$$
\begin{gathered}
L\left\{D_{t}^{-n} f(t)\right\}=s^{-n} F(s) \\
L\left\{D_{t}^{n} f(t)\right\}=s^{n} F(s)
\end{gathered}
$$

where $F(s)=L\{f(t)\}$ and $s$ is the Laplace complex variable. The Fourier transform can be obtained by replacing $s$ by $j \omega$ in the Laplace transform and the equivalent frequencydomain expressions are:

$$
\begin{aligned}
& \frac{1}{(j \omega)^{n}}=\frac{1}{\omega^{n}}\left(\cos \frac{n \pi}{2}-j \sin \frac{n \pi}{2}\right) \\
& (j \omega)^{n}=\omega^{n}\left(\cos \frac{n \pi}{2}+j \sin \frac{n \pi}{2}\right)
\end{aligned}
$$

Thus, the modulus and the argument of the FO terms are given by:

$$
\begin{gathered}
\text { Modulus }(\mathrm{dB})=20 \log \left|(j \omega)^{\mp n}\right|=\mp 20 n \log |\omega| \\
\text { Phase }(\mathrm{rad})=\arg \left((j \omega)^{\mp n}\right)=\mp n \frac{\pi}{2}
\end{gathered}
$$

resulting in a straight line with a slope of $\mp 20 n$ passing through $0 \mathrm{~dB}$ for $\omega=1$ for the magnitude ( $\mathrm{dB}$ vs. logfrequency), respectively a horizontal line, thus independent with frequency, with value $\mp n \frac{\pi}{2}$ for the phase ( $\operatorname{rad} v s$. logfrequency). The respective sketches are given in figure 8 .

\section{Principles of Compartmental Fractional Derivative Models}

In this section a two compartmental fractional derivative model is discussed. The basic idea behind this model can be used to model diffusion processes in the human body by a multi-compartmental model.

The model is formulated so that the mass balance is 

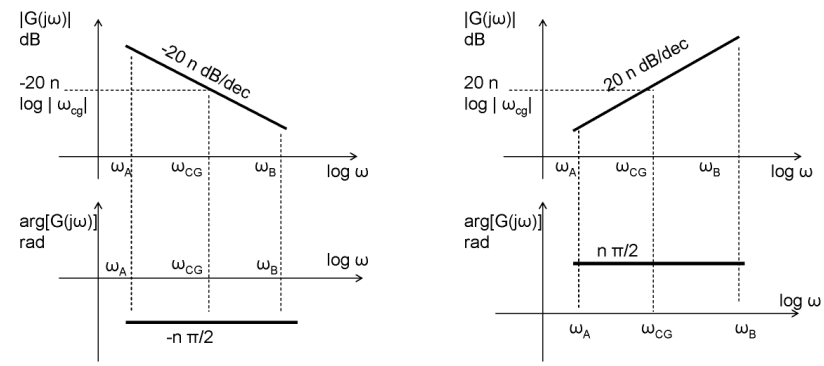

Fig. 8. Sketch representation of the FO integral and derivator operators in frequency domain, by means of the Bode plots (magnitude above and phase bellow).

preserved. In figure 9, we see a conceptual schematic of a model with two compartments. Assume that $q_{i}(t)=v_{i} c_{i}$, for $i=1,2$ denote the amount of a drug in a specific compartment. Here $c_{i}$ is the concentration of a drug and $v_{i}$ is the volume of the $i$-th compartment and $K_{i j}$ is the fractional rate of transfer from compartment $i$ to compartment $j$.

The first compartment represents the place where the drug is applied i.e. muscle, subcutaneous tissue or digestive tract. The second compartment represents the plasma or any other region in the body where the kinetics of the drug are uniform. Traditionally, the two compartments are described by a system of differential equations of integer order.

$$
\begin{aligned}
& \frac{d q_{1}(t)}{d t}=K_{21} q_{2}(t)-K_{12} q_{1}(t)-K_{01} q_{1}(t) \\
& \frac{d q_{2}(t)}{d t}=K_{12} q_{1}(t)-K_{21} q_{2}(t)-K_{02} q_{2}(t)
\end{aligned}
$$

Recently, the fractional-order models seem to better suit the dynamics of biological systems than other integer models. A simple model of a two-compartmental system is then given by the following equations:

$$
\begin{gathered}
\tau_{1}^{n_{1}-1}{ }_{0} D_{t}^{n_{1}} q_{1}(t)=-K_{12} q_{1}(t), \\
\tau_{2}^{n_{2}-1}{ }_{0} D_{t}^{n_{2}} q_{2}(t)=K_{12} q_{1}(t)-K_{02} q_{2}(t),
\end{gathered}
$$

where we assumed $K_{01}=0, K_{21}=0$ and with the initial conditions $q_{1}(0)=$ dose, and $q_{2}(0)=0$. In these equations $\tau_{1}$ and $\tau_{2}$ are time constants which represent the speed of diffusion, while $n_{1}$ and $n_{2}$ represent a non-integer between

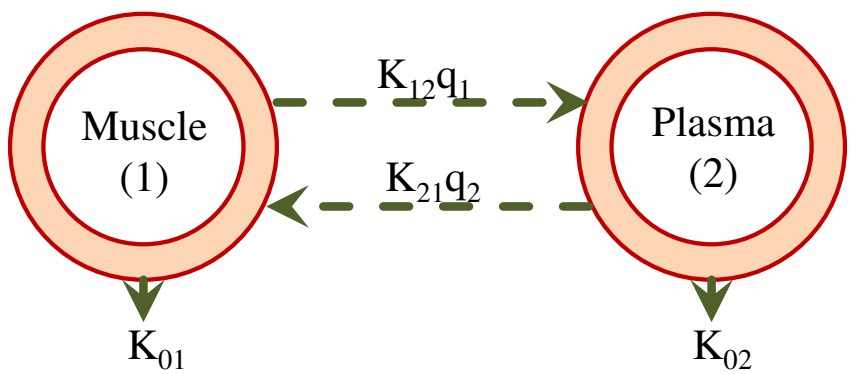

Fig. 9. Conceptual schematic of a two-compartment model.
0 and 1 and characterize the type of diffusion (sub-, super-, etc).

\section{GRAND CHALLENGES}

Several grand challenges are encountered in the development of the proposed analgesic biosensor. We discuss the major challenges in the remainder of this section.

To be able to control the level of analgesia in the unconscious patient, we apply a MPC strategy. This strategy needs a reliable model of the process that it needs to control. In traditional, non-human, systems, this model identification is done by sending excitation signals into the system and analyzing the corresponding output signals of the open-loop system. However, as we are dealing with patients, it is not possible to apply here the same strategy. Output signals to analyze are available only after the nurse administers a certain amount of analgesic drug. As the nurse only administers this drug after examination of the patient, this is no longer an open-loop system and system identification can be compromised by this.

Another difficulty in the model identification is the fact that every person reacts differently to a certain amount or combination of drugs. Therefore, every model differs for every patient i.e. inter-patient variability.

Moreover, the conditions inside the body of every patient are changing as a result of accumulated drug effect i.e. intrapatient variability. The parameters of the patient model need to be updated regularly.

The pain sensor is supposed to measure a pain signal in an unconscious patient. However, there is no reliable way to validate the pain sensor once it is developed as the patient is the only one who can feel the pain but he is not able to indicate it anymore because he is unconscious.

Even if you can objectively proof that the pain sensor picks up a pain signal in one patient, it is not certain that the sensor will have the same result in a different patient as the pain threshold for one person can be completely different for each person. This is the result of the inter- and intra-patient variabilities that pose an extra challenge on the development of the pain sensor.

Another major challenge in this research direction is the fact that a combination of drugs is administered to the patient. Therefore, it is difficult to completely separate and validate the effect of the analgesic drug.

\section{CONCLUSIONS}

This paper proposes an early dawn pain sensor to measure analgesia level in unconscious patients. The proposed sensor can be used in combination with a model-based predictive control strategy to control the level of anesthesia in an unconscious patient. To model the diffusion process in the human body a fractional-order impedance model is applied. The coinciding challenges in this research direction include identification of the patient model, validation of the pain sensor and validation of the effect of the analgesic drug. 


\section{ACKNOWLEDGMENT}

C. M. Ionescu acknowledges the Flanders Research Center (FWO) for its financial support.

\section{REFERENCES}

[1] J. M. Bailey and W. M. Haddad, "Drug dosing control in clinical pharmacology”, IEEE Control Systems Magazine, vol. 25, no. 2, pp. 35-51, Apr. 2005.

[2] W. M. Haddad, T. Hayakawa and J. M. Bailey, "Nonlinear adaptive control for intensive care unit sedation and operating room hypnosis", American Control Conference, vol. 2, pp. 1808-1813, 2003.

[3] D. O'Hara, D. Bogen and A. Noordergraaf, "The use of computers for controlling the delivery of anesthesia", Anesthesiology, vol. 77, pp. 563-581, 1992.

[4] S. G. Greenhow, D. A. Linkens and A. J. Asbury, "Pilot study of an expert system adviser for controlling general anesthesia", British Journal of Anesthesia, vol. 71, pp. 359-365, 1993.

[5] M. Struys, H. Vereecke, A. Moerman, E. W. Jensen, D. Verhaeghen, N. De Neve, F. Dumortier and E. Mortier, "Ability of the bispectral index, autoregressive modelling with exogenous input-derived auditory evoked potentials, and predicted propofol concentrations to measure patient responsiveness during anesthesia with propofol and remifentanil", Anesthesiology, vol. 99, pp. 802-814, 2003.

[6] R. B. Northrop, Endogenous and Exogenous Regulation and Control of Physiological Systems. Boca Raton, FL:CRC, 2000.

[7] R. De Keyser, "Model based predictive control", UNESCO Encyclopedia Life Support System, vol. 6.43.16.1, pp. 802-812, 2003.

[8] J. Kress, A. Pohlman and J. Hall, "Sedation and analgesia in the intensive care unit", American Journal of Respiratory and Critical Care Medicine, vol. 166, pp. 1024-1028, 2002.

[9] O. Steen-Knudsen, Biological Membranes. Theory of transport, potentials and electric impulses. Cambridge: Cambridge University Press, 2002.
[10] J. M. Berg, ed., Biochemistry, 6th ed. New York City, NY: W.H. Freeman and Company, 2002.

[11] R. De Keyser and A. Van Cauwenberghe, "A self-tuning multistep predictor application", Automatica, vol. 17, pp. 167-174, 1981.

[12] T. M. Hemmerling, E. Salhab, G. Aoun , S.Charabati and P. Mathieu , "The Analgoscore: a novel score to monitor intraoperative pain and its use for Remifentanil closed loop application", IEEE Proceedings Cat., ref nr. 1-4244-0991-8/07, pp. 1494-1499, 2007.

[13] C. N. Sessler, M. Gosnell, M. J. Grap, G. T. Brophy, P. V. O’Neal, E. Tesoro and R.K. Elswick, "A new Agitation-Sedation Scale for critically ill patients: development and testing of validity and interrater reliability", American Journal of Respiratory and Critical Care Medicine, vol. 161, pp. A506, 2000.

[14] P. S. Glass, M. Bloom, L. Kearse, C. Rosow, P. Sebel and P. Manberg, "Bispectral analysis measures sedation and memory effects of propofol, midazolam, isoflurane, and alfentanil in healthy volunteers", Anesthesiology, vol. 86, pp. 836-847, 1997.

[15] G. A. Losa, D. Merlini, T. F. Nonnenmacher and E. R. Weibel, Fractals in Biology and Medicine, vol IV. Berlin: Birkhaser, 2005.

[16] A. Oustaloup, La derivation non entiere. Hermes, Paris, 1995. (in French)

[17] C. M. Ionescu, J. T. Machado and R. De Keyser, "Modeling of the Lung Impedance using a Fractional Order Ladder Network with Constant Phase Elements", IEEE Transactions on Biomedical Circuits and Systems, vol. 5, no. 1, pp. 83-89, 2011.

[18] B. Lundstrom, M. Higgs, W. Spain and A. Fairhall, "Fractional differentiation by neocortical pyramidal neurons", Nature Neuroscience, vol. 11, pp. 1135-1342, 2008.

[19] C. M. Ionescu, "Phase constancy in a ladder model of neural dynamics", IEEE Transactions on Systems, Man and Cybernetics, part A: Systems and Humans, vol. 42, no. 6, 2012.

[20] A. Dokoumetzidis, R. Magin and P. Macheras, "A commentary on fractionalization of multi-compartmental models", Journal of Pharmacokinetics \& Pharmacodynamics, vol. 37, no. 2, pp. 203-207, April 2010. 